\title{
Integrin-linked kinase mediates CTGF-induced epithelial to mesenchymal transition in alveolar type II epithelial cells
}

\author{
Mitra Shafieian', Shaoyi Chen² and Shu Wu²
}

\begin{abstract}
BACKGROUND: Overexpression of connective tissue growth factor (CTGF) in alveolar type II epithelial (AT II) cells disrupts alveolar structure, causes interstitial fibrosis, and upregulates integrin-linked kinase (ILK). Whether CTGF-ILK signaling induces epithelial to mesenchymal transition (EMT) in AT II cells is unknown.
\end{abstract}

METHODS: Transgenic mice with targeted overexpression of CTGF in AT II cells were generated utilizing the surfactant protein C (SP-C) gene promoter and doxycycline-inducible system. AT II cells were isolated from 4-wk-old CTGF-overexpressing (CTGF+) mice and control littermates, and cultured on Matrigel. Cells were transfected with ILK siRNA, and cell morphology and expression of cell differentiation markers were analyzed.

RESULTS: The AT II cells from the control lungs grew in clusters and formed alveolar-like cysts and expressed SP-C. In contrast, the cells from CTGF+ lungs were spread and failed to form alveolar-like cysts. These cells expressed higher levels of CTGF, a smooth muscle actin (a-SMA), fibronectin and vimentin, the mesenchymal markers, suggesting EMT-like changes. Transfection with ILK siRNA not only dramatically attenuated ILK expression, but also decreased a-SMA expression as well as reversed cell morphological changes in CTGF+ AT II cells.

CONCLUSION: Overexpression of CTGF induces EMT in mouse primary AT II cells and this is mediated by ILK.

\section{INTRODUCTION}

Pulmonary fibrosis occurs in a variety of clinical sittings and affects patients of all ages. Interstitial lung fibrosis is one of pathological hallmarks of bronchopulmonary dysplasia, also known as chronic lung disease of premature infants (1). Idiopathic pulmonary fibrosis is the most common and severe form of interstitial lung disorder in adult patients (2). Pulmonary fibrosis causes destruction of alveolar structure and respiratory function, and progressive fibrosis is a significant cause of morbidity and mortality and there is no effective therapy. Although the histopathologic pattern of lung fibrosis has been long recognized to consist of nonspecific interstitial fibrosis, resulting from fibroblast proliferation, myofibroblast differentiation and excessive deposition of extracellular matrix (ECM), the specific molecular and cellular mechanisms are unknown (2).
The key effector cell in fibrogenesis is the myofibroblast which is responsible for the synthesis and deposition of ECM and the resultant structural remodeling that leads to the loss of alveolar structure and end-stage lung disease (3). These myofibroblasts have been traditionally thought to be differentiated directly from resident alveolar fibroblasts under the influence of the profibrotic microenvironment $(2,3)$. However, novel concepts of fibrogenesis indicate a role of epithelialmesenchymal transition (EMT) as a source of myofibroblasts following epithelial injury $(4,5)$. A number of growth factors have been implicated in the pathogenesis of lung fibrosis, and transforming growth factor $\beta$ (TGF- $\beta$ ) is one of the most critical factors implicated (6-8). TGF- $\beta$ has the ability to stimulate both fibroblast to myofibroblast differentiation and EMT. Indeed, strategies to block the biological activity of TGF- $\beta$, including neutralizing antibodies, soluble receptors, and pharmacological inhibitors of Smad proteins, have been used in animal models with varying degrees of attenuation of lung fibrosis (6,9-11). However, given the exceptionally broad range of biological activity ascribed to TGF- $\beta$ and its fundamental physiological roles, nonselective TGF- $\beta$ blockade could have undesired consequences. Complete abrogation of TGF- $\beta$ signaling could lead to loss of immune tolerance, spontaneous autoimmunity and defective tissue repair (12). Therefore, identification of novel mediators and mechanisms will not only enhance our understanding of the pathogenesis of lung fibrosis but also provide effective targets for treating this devastating disease.

Connective tissue growth factor (CTGF), a multimodular matrix-associated protein, is thought to be the downstream mediator of TGF- $\beta$ with a particular role in stimulating fibroblast proliferation, fibroblast to myofibroblast differentiation and ECM deposition (13-15). Expression of CTGF is upregulated by TGF- $\beta$ and anti-CTGF antibody blocks TGF$\beta$-induced fibrogenic effects (16-18). Upon stimulation, CTGF is secreted into the extracellular environment where it interacts with distinct cell surface receptors, growth factors and ECM. The principal CTGF receptor is the heterodimeric cell surface integrin complexes (19-21), while integrin-linked kinase (ILK) is a key mediator of integrin signaling that interacts with the cytoplasmic domain of $\beta$ integrins (22-24). Most 
importantly, ILK signaling plays a critical role in EMT under many pathological conditions (25-27).

There is increasing evidence that CTGF plays an important role in lung fibrosis. Increased CTGF expression is detected in patients with lung fibrosis and bronchopulmonary dysplasia (28-30). Transgenic overexpression of CTGF in fibroblasts resulted in systemic fibrosis, including the lung (31). Furthermore, in vivo and in vitro studies have shown that expression of CTGF in lung epithelial cells or treatment with exogenous CTGF induces EMT-like changes (32). Previous studies from our laboratory have demonstrated that targeted overexpression of CTGF in alveolar type II epithelial (AT II) cells disrupts alveolar structure and induces interstitial fibrosis, and these pathological changes were associated with activation of ILK signaling (33). It is known that ILK mediates CTGF-induced EMT in renal tubular cells (34). However, whether ILK mediates CTGFinduced EMT in AT II cells is unknown.

To test the hypothesis that overexpression of CTGF in AT II cells induces EMT and this is mediated by ILK, we cultured primary AT II cells that were isolated from transgenic mice with targeted overexpression of CTGF in these cells and performed transfection with ILK siRNA. We demonstrated that AT II cells with overexpression of CTGF undergo EMT-like changes and knockdown of ILK gene attenuates this process.

\section{RESULTS}

\section{Overexpression of CTGF Induces EMT-Like Phenotype in AT II \\ Cells}

After being cultured for $96 \mathrm{~h}$, the control cells grew in clusters and formed alveolar-like cysts (Figure 1a). In contrast, the a

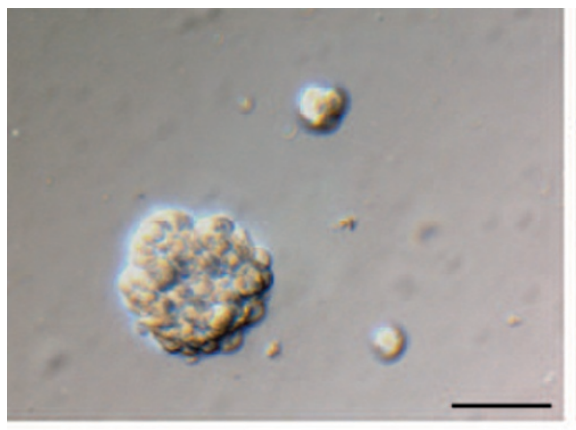

C

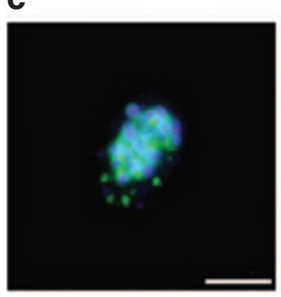

e

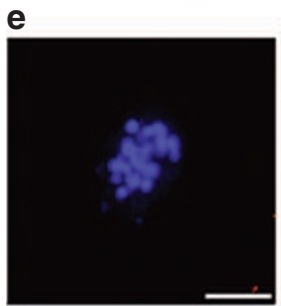

g

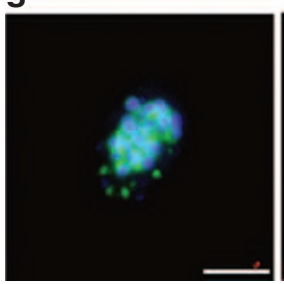

d

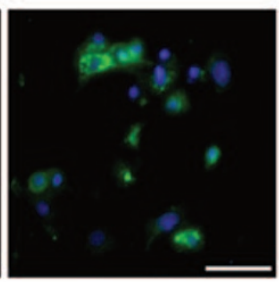

f

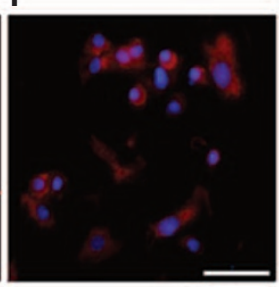

h

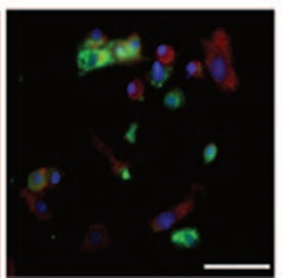

b

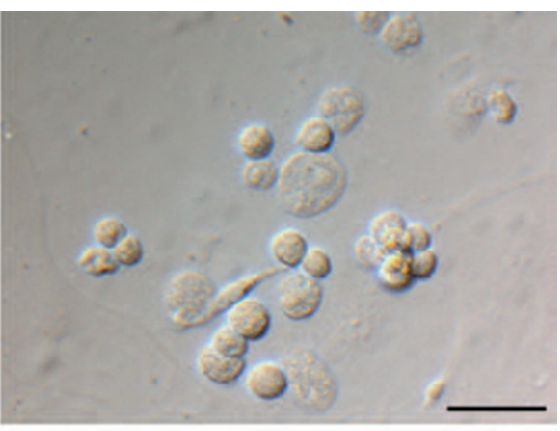

i

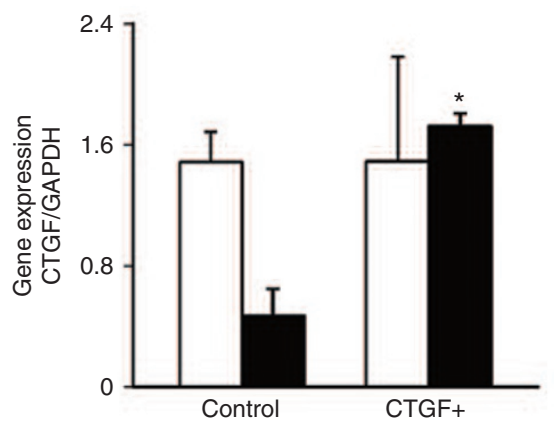

Figure 1. Overexpression of connective tissue growth factor (CTGF) in AT II cells induces EMT. Cell morphology of AT II cells from (a) control and (b) CTGF+ mice after being cultured for $96 \mathrm{~h}$ (scale bar $=50 \mu \mathrm{m}$ ). The control cells grew in clusters and formed alveolar-like cysts. The CTGF+ cells were large, spread and some of them were spindle shaped. Double immunofluorescence staining for surfactant protein C (SP-C) (green), CTGF (red), DAPI nuclear stain (blue), and merge (yellow) showed that $(\mathbf{c}, \mathbf{e}, \mathbf{g})$ control AT II cells are positive for SP-C and negative for CTGF and (d,f,h) CTGF+ AT II cells are positive for both SP-C and CTGF (scale bar $=50 \mu \mathrm{m}$ ). (i) qRT-PCR demonstrated that control AT Il cells express high level of SP-C and low level of CTGF, and CTGF+ AT II cells express SP-C and CTGF (white bar: SP-C, black bar: CTGF). $n=3 /$ group. ${ }^{*} P<0.001$ vs. control CTGF gene expression. 


\section{Articles | Shafieian et al.}

AT II cells from CTGF mice were spread and failed to form alveolar-like cysts (Figure 1b). Double immunofluorescence staining demonstrated that the control cells express SP-C not CTGF (Figure 1c,e,g), but the AT II cells from CTGF+ mice expressed both SP-C and CTGF (Figure 1d,f,h). Quantitative reverse-transcriptase (qRT)-PCR confirmed that control AT II cells express high a level of $S P-C$ gene and a low level of $C T G F$ gene, but CTGF AT II cells expressed SP-C and high level of CTGF genes (Figure 1i). Together, these results indicate that transgenic overexpression of CTGF in AT II cells can be maintained in culture and this lead to EMT-like phenotype.

\section{Overexpression of CTGF Induces $\alpha$-SMA Expression and Upregulates ILK in AT II Cells}

To further confirm EMT, we assessed $\alpha$ smooth muscle actin $(\alpha$-SMA) expression in AT II cells. Double immunofluorescence staining demonstrated that CTGF overexpressing AT II cells also express $\alpha$-SMA which was not detected in control AT II cells (Figure 2). We next assessed whether overexpression of CTGF in AT II cells affects ILK expression. Double immunofluorescence staining showed that CTGF overexpressing AT II cells express ILK and this was not detected in control AT II cells (Figure 3). These data highlight that overexpression of CTGF in AT II cells induces a molecular change of EMT and this is associated with increased ILK.

\section{Knockdown of ILK Attenuates CTGF-Induced EMT in AT II Cells}

We further investigated the role of ILK in CTGF-induced EMT utilizing a loss-of function approach. Transfection of AT II cells with ILK-specific siRNAs significantly decreased ILK gene and protein expression in both control and CTGF AT II cells (Figure 4). As a consequence, this leaded to a drastic decrease a

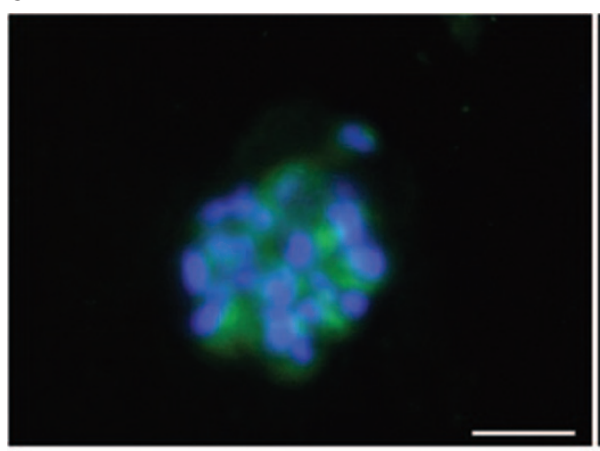

C

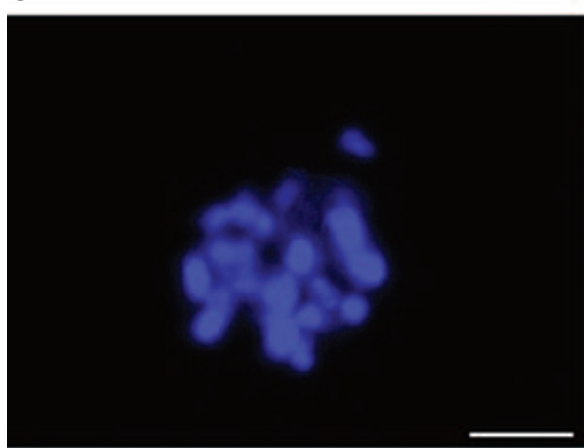

e

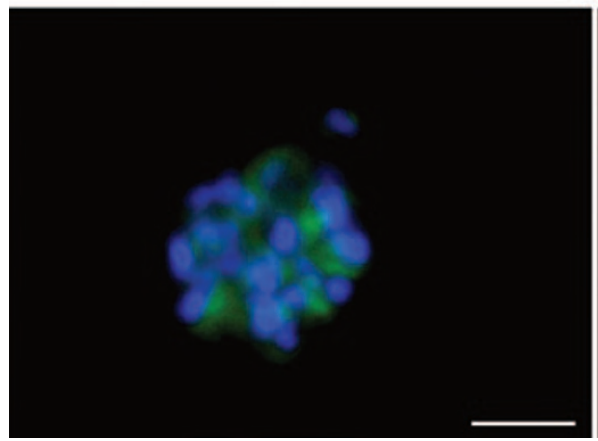

b

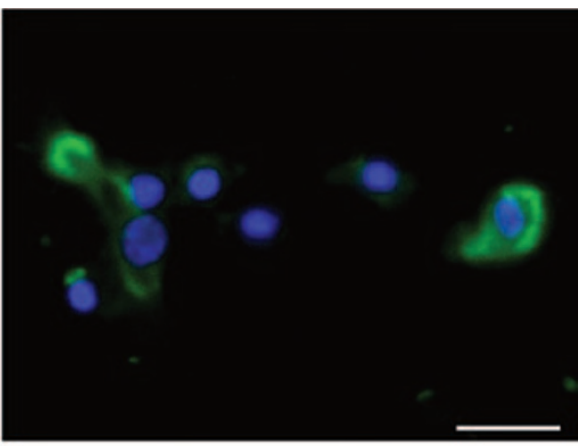

d

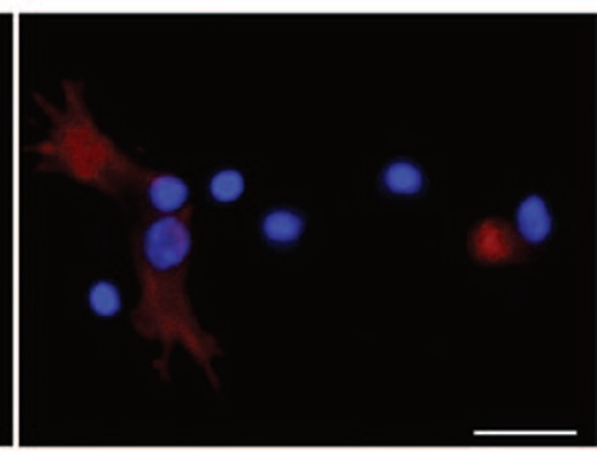

f

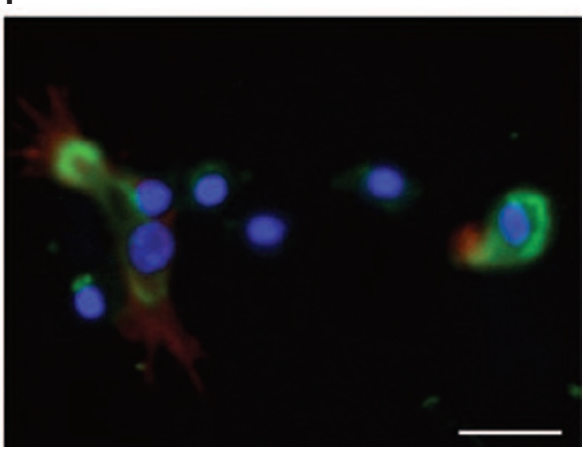

Figure 2. Overexpression of connective tissue growth factor (CTGF) in AT II cells increases $\alpha$ smooth muscle actin ( $\alpha$-SMA) expression. Double immunofluorescence staining for surfactant protein C (SP-C) (green), $\alpha$-SMA (red), DAPI nuclear stain (blue), and merge (yellow) showed that $(\mathbf{a}, \mathbf{c}, \mathbf{e})$ control AT II cells express SP-C but not $\alpha$-SMA. However, some of $(\mathbf{b}, \mathbf{d}, \mathbf{f})$ CTGF+ AT II cells coexpress SP-C and $\alpha-S M A$. Scale bar $=50 \mu m$. 
b
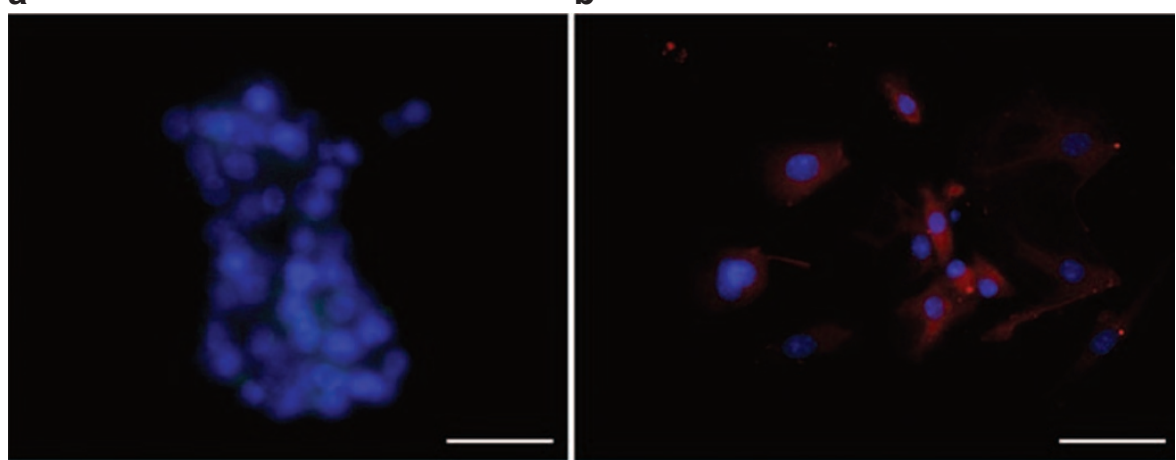

C

d
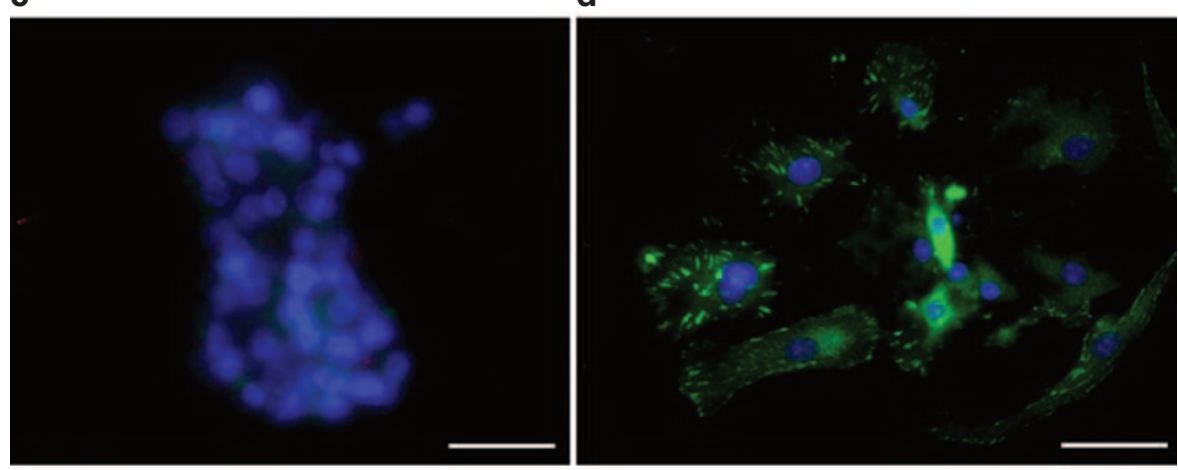

e

f
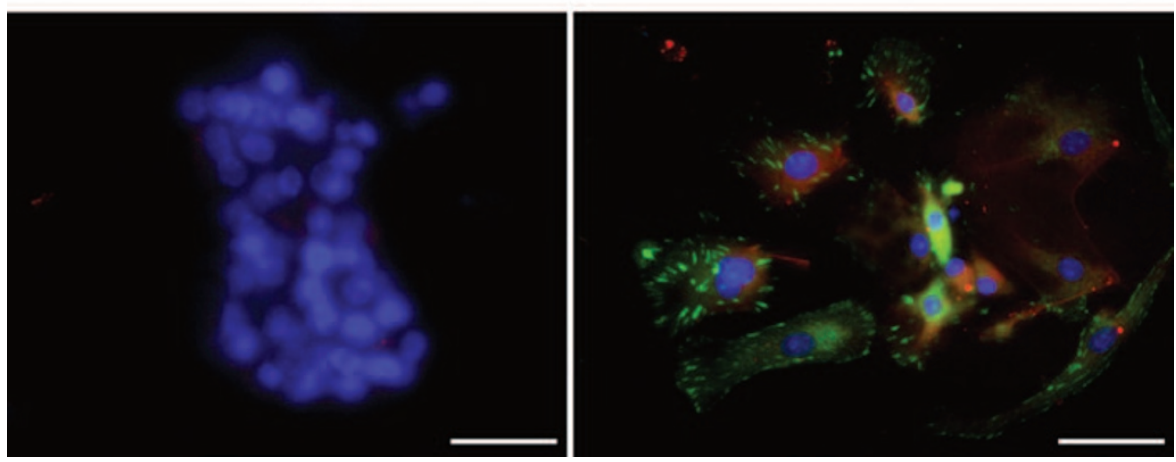

Figure 3. Overexpression of connective tissue growth factor (CTGF) in AT Il cells induces integrin-linked kinase (ILK). Double immunofluorescence staining for CTGF (red), ILK (green), DAPI nuclear stain (blue) and merge showed that $(\mathbf{a}, \mathbf{c}, \mathbf{e})$ control AT II cells do not express CTGF and ILK. However, $(\mathbf{b}, \mathbf{d}, \mathbf{f})$ some of CTGF+ AT II cells co-expressed CTGF and ILK. Scale bar $=50 \mu \mathrm{m}$.

of $\alpha$-SMA gene and protein expression in CTGF+ cells to the level of control cells (Figure 5). The ILK siRNA-treated CTGF+ cells also had a similar morphology to the control cells (Figure 5). We also assessed expression of additional two mesenchymal markers, vimentin and fibronectin, and found that ILK siRNA significantly decreased their gene expression in CTGF+ cells to the levels in control cells (Figure 6). These data suggest that ILK mediates CTGF-induced EMT in AT II cells.

\section{DISCUSSION}

In this study, we demonstrate that primary AT II cells from mice with targeted overexpression of CTGF under the control of SP-C gene promoter undergo EMT-like changes in culture and these were associated with upregulation of ILK. Most importantly, we showed the evidence that ILK mediates CTGF-induced EMT-like phenotype in AT II cells. These data highlight a novel autocrine mechanism by which CTGF-ILK signaling regulates EMT in AT II cells.

CTGF is a multimodular matrix-associated protein that plays an important role in cell proliferation, differentiation, cell survival, and ECM production during tissue development and injury repair. Recent advances in genetic modification technology have provided us with the ability to highlight the cell-specific role of CTGF in lung development and pathology. Ivkovic et al. (35) have shown that global knockout of CTGF results in respiratory failure and immediate death in newborn mice suggesting that endogenous CTGF in lung cells are important for embryonic lung development and newborn lung function. Sonnylal et al. $(31,32)$ demonstrated that targeted overexpression of CTGF in lung fibroblasts induces fibrosis which affects both lung fibroblasts and epithelial cells indicating that fibroblast-derived CTGF results in lung fibrosis via both autocrine 


\section{Articles | Shafieian et al.}
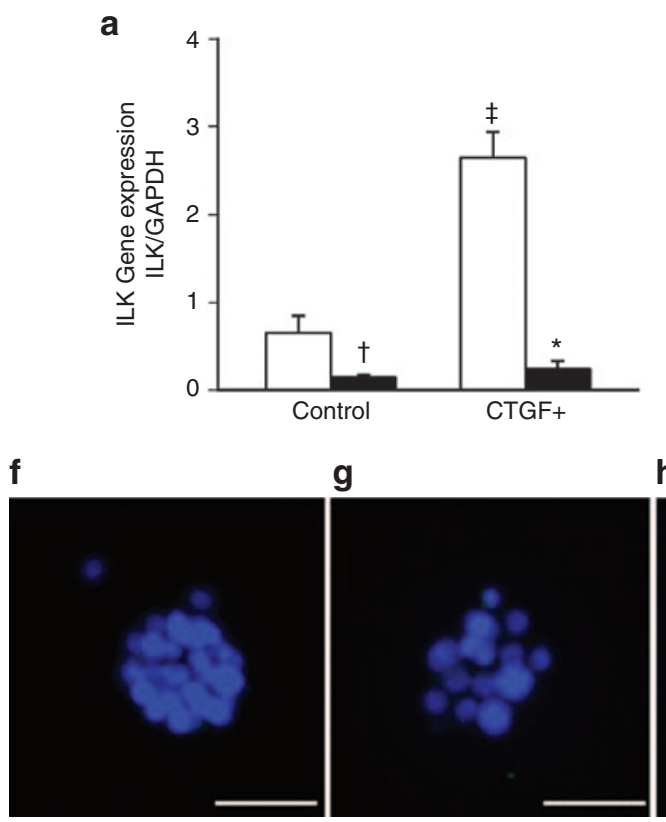

$\mathbf{h}$
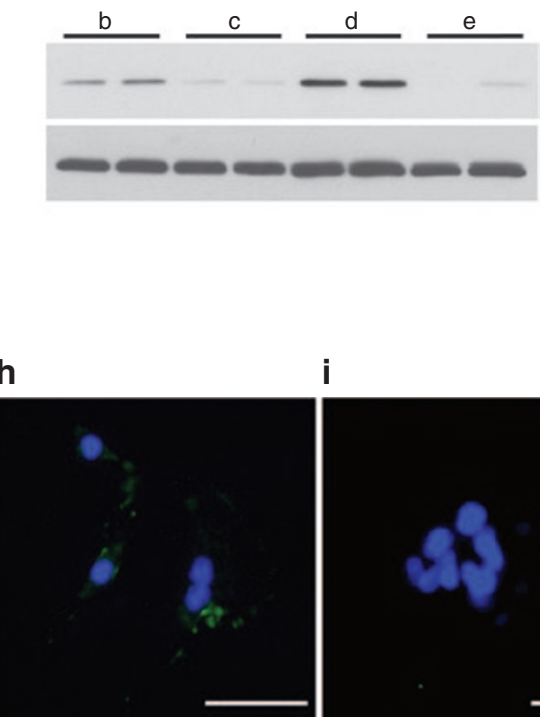

i

Figure 4. Integrin-linked kinase (ILK) siRNA knockdown of ILK in AT II cells. (a) qRT-PCR demonstrated that transfection with ILK siRNA significantly knockdown ILK gene expression in control and CTGF+ AT Il cells as compared to negative control (NC) siRNA transfection (white bar: NC siRNA; black bar: ILK siRNA). $n=3$ /group. ${ }^{\dagger} P<0.05$ vs. NC siRNA-treated control cells; ${ }^{\ddagger} P<0.001$ vs. NC siRNA-treated control cells; ${ }^{*} P<0.001$ vs. NC siRNA-treated CTGF+ cells. Representative Western blot images demonstrated drastic decreases in ILK expression in ILK siRNA-treated control and CTGF+ AT II cells. (b) NC siRNA transfected control cells; (c) ILK siRNA transfected control cells; (d) NC siRNA transfected CTGF+ cells; and (e) ILK siRNA transfected CTGF+ cells. Immunofluorescence staining for ILK (green) and nuclear DAPI staining (blue) demonstrated a drastic decrease in ILK expression in CTGF+ AT II cells. (f) NC siRNA transfected control cells; (g) ILK siRNA transfected control cells; (h) NC siRNA transfected CTGF+ cells; and (i) ILK siRNA transfected CTGF+ cells. Scale bar $=50 \mu \mathrm{m}$. CTGF, connective tissue growth factor.

and paracrine mechanisms. Our previous studies showed that targeted overexpression of CTGF in AT II cells disrupts alveolar development and induces lung fibrosis (33). Our unpublished data also showed that there are increased CTGF concentrations in bronchoalveolar lavage fluid and serum in mice with overexpression of CTGF in AT II cells. Taken together, these results suggest that both epithelial and mesenchymal CTGF are critical for lung development and lung fibrosis.

It is suggested that repeated injury to the alveolar epithelium sets up a cascade of dysregulated epithelial-mesenchymal crosstalk, through the release of growth factors, matrix metalloproteinase, cytokines, and chemokines that in turn leads to mesenchymal cell activation/proliferation and fibrogenesis $(36,37)$. However, increasing evidence indicates that alveolar epithelial cells have the ability to undergo EMT upon injury and exposure to certain stimuli, and this contributes to pulmonary fibrosis $(4,5)$. During EMT, epithelial cells loss apicalbasal polarity, basement membrane attachment and cell-cell contact, and gain mesenchymal cell features and behavior. Many growth factors and signaling molecules have been implicated in this process, and TGF- $\beta$ is one of the critical factors that have been extensively studied $(38,39)$. Although CTGF is a downstream mediator of many of TGF- $\beta$ fibrogenic actions, the role of CTGF in EMT, particular in AT II cell EMT is poorly understood.

In this study, we isolated AT II cells from our transgenic mice with targeted overexpression of CTGF in these cells and cultured them on Matrigel. This culture system has the ability to maintain certain of AT II cell behaviors such as forming alveolar-like cysts and expressing surfactant proteins $(33,40)$. We showed that AT II cells with overexpression of CTGF are larger, some of them are in spindle shape and do not form alveolar-like cyst. Furthermore, these cells expressed mesenchymal markers such as $\alpha$-SMA, fibronectin, and vimentin, suggesting EMT-like changes. Sonnylal et al., have demonstrated that CTGF causes EMT-like cell fate in vivo and in vitro in the lung $(31,32)$. They used transgenic mice with mesenchymal overexpression of CTGF driven by the collagen $1 \mathrm{a} 2$ promoter as well as lung epithelial cells lines A549 and SV40-T2. The in vivo studies revealed that lung fibrosis is associated with a marked increase in cells coexpressing epithelial and mesenchymal markers suggesting EMT. In vitro, they showed that adenoviral mediated overexpression of CTGF as well as treatment with recombinant CTGF induces EMT-like changes in lung epithelial cells. Taken together, these studies provided important evidence that fibroblast-CTGF and epithelial-CTGF via autocrine and paracrine mechanisms induces lung fibrosis and EMT.

The important finding of this study is that we identified a novel signaling pathway, the CTGF-ILK pathway regulates EMT in AT II cells. ILK is an intracellular serine/threonine protein kinase that interacts with the cytoplasmic domains of integrins and numerous cytoskeleton-associated proteins. CTGF has the ability to bind to cell surface integrins, thus activating ILK signaling. ILK has been identified as a regulator of EMT in several epithelia e.g., renal, ovary, and lens and mammary glands (25-27). We showed that AT II cells with overexpression 
a

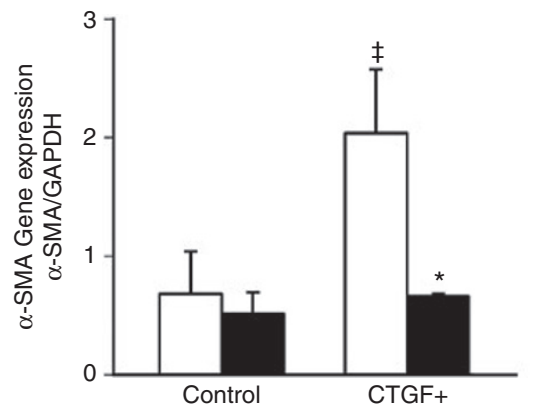

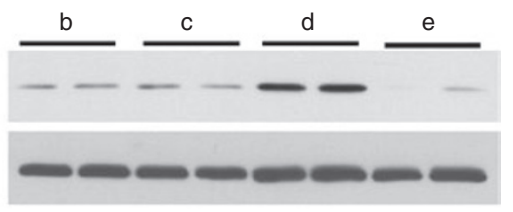

h

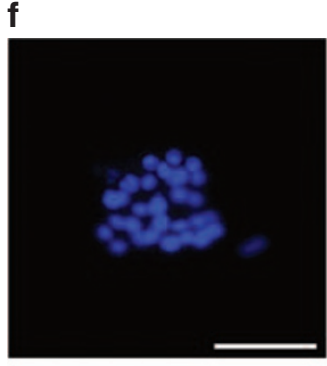

g
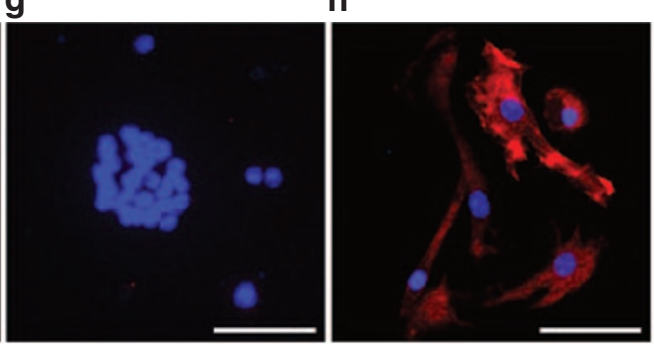

i

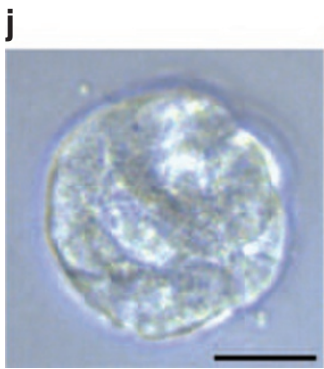

k

I

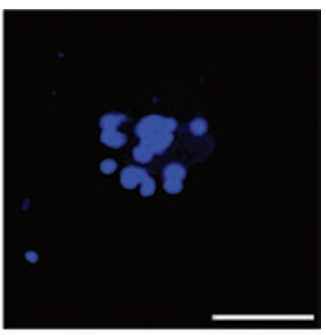

m
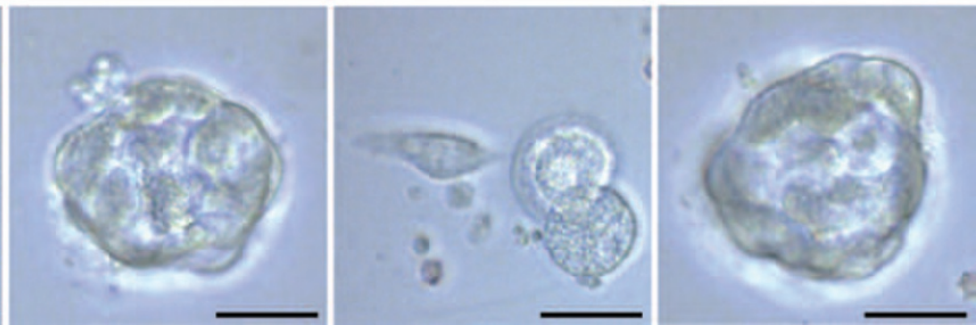

Figure 5. Transfection with integrin-linked kinase (ILK) siRNA decreases $\alpha$ smooth muscle actin ( $\alpha$-SMA) expression. (a) qRT-PCR demonstrated that transfection with ILK siRNA significantly decreased $\alpha$-SMA gene expression in CTGF+ AT II cells as compared to negative control (NC) siRNA transfection (white bar: NC siRNA; black bar: ILK siRNA). $n=3$ /group. ${ }^{\ddagger} P<0.001$ vs. NC siRNA-treated control cells; ${ }^{*} P<0.001$ vs. NC siRNA-treated CTGF+ cells. Representative Western blot images demonstrated drastic decreases in $\alpha$-SMA expression in ILK siRNA-treated CTGF+ AT II cells. (b) NC siRNA transfected control cells; (c) ILK siRNA transfected control cells; (d) NC siRNA transfected CTGF+ cells and (e) ILK siRNA transfected CTGF+ cells. Immunofluorescence staining for $\alpha$-SMA (red) and nuclear DAPI staining (blue) demonstrated a drastic decrease in $\alpha$-SMA expression in CTGF+ AT II cells. (f) NC siRNA transfected control cells; (g) ILK siRNA transfected control cells; (h) NC siRNA transfected CTGF+ cells; and (i) ILK siRNA transfected CTGF+ cells. Cell morphology from (j) NC siRNA-treated control and (i) CTGF+ cells, and (k) ILK siRNA-treated control and (m) CTGF+ cells. Scale bar $=50 \mu \mathrm{m}$. CTGF, connective tissue growth factor.
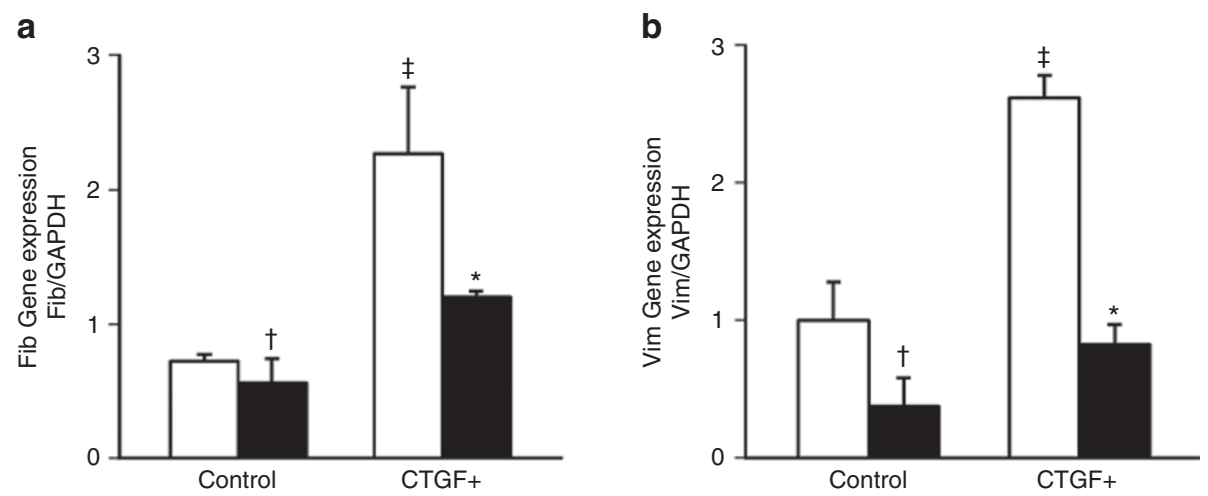

Figure 6. Transfection with integrin-linked kinase (ILK) siRNA decreases fibronectin and vimentin gene expression. qRT-PCR demonstrated that transfection with ILK siRNA significantly decreased (a) fibronectin (Fib) gene and (b) vimentin (Vim) gene expression in CTGF+ AT II cells as compared negative control (NC) siRNA transfection (white bar: NC siRNA; black bar: ILK siRNA). $n=3 /$ group. ${ }^{\dagger} P<0.05$ vs. NC siRNA treated control cells; ${ }^{\ddagger} P<0.001$ vs. NC siRNA treated control cells; ${ }^{*} P<0.001$ vs. NC siRNA treated CTGF+ cells. CTGF, connective tissue growth factor. 
of CTGF express high levels of ILK gene and protein. However, knockdown of ILK by siRNA drastically decreased expression of fibronectin, vimentin and $\alpha$-SMA, key mesenchymal markers and known CTGF target genes. Furthermore, knockdown of ILK protected AT II cells undergo EMT-like phenotype. These data indicate that overexpression of CTGF-induced EMT in AT II cells is at least in part mediated by ILK.

Besides ILK, many other intracellular kinases such as focal adhesion kinase and extracellular signal-regulated kinase (ERK) also mediate integrin signaling (22). Moreover, CTGF can also activate p42/44 mitogen-activated protein kinase (MAPK) as well as Smad2/3 signaling via integrin-independent mechanisms (14). Previous studies have shown that overexpression of CTGF in skin and lung fibroblasts activates MAPK and ERK signaling in the adjacent epithelial cells that undergo EMT-like changes $(31,32)$. It is unclear whether activation of MAPK and ERK in these cells via integrin-dependent signaling. Given the complexity of CTGF signaling network and its biological significance in lung fibrosis, further investigation is needed to delineate the role of these kinases in mediating CTGF-integrin signaling in alveolar epithelial cells that may contributes to our overall understanding in the mechanisms by which CTGF-induces EMT in lung fibrosis.

In summary, this in vitro study demonstrates that transgenic overexpression of CTGF induces EMT-like phenotype in AT II cells. Furthermore, knockdown of ILK attenuates this process, thus suggesting an autocrine mechanism by which CTGF-ILK signaling regulates EMT in AT II cells. Although there are increasing reports on the critical role of EMT in lung fibrosis in animal models and in cell cultures, little is known about the role of EMT in patients developing lung fibrosis. Much work needs to be done to elucidate the potential autocrine and paracrine mechanisms by which CTGF-ILK signaling contributes to EMT and lung fibrosis in human. This may help to generate novel therapies by targeting CTGF-ILK signaling to prevent and treat lung fibrosis, a devastating disorder that affects patients from neonates to adults.

\section{METHODS}

\section{CTGF Transgenic Mice}

The study protocols were reviewed and approved by the Animal Care and Use Committee at the University of Miami (Miami, FL). The conditional and tissue-specific overexpression of CTGF was achieved by mating two lines of transgenic mice, the SP-C-rtTA mice (line 2), bearing the reverse tetracycline responsive transactivator $(\mathrm{rtTA})$ under the control of the $3.7-\mathrm{kb}$ rat SP-C gene promoter and the TetO-CTGF mice, containing tetracycline operator (TetO) and minimal CMV promoter and CTGF transgene, as we have previously described (33). The SP-C-rtTA mice were provided by Dr Jeffrey Whitsett (Cincinnati Children's Hospital, Cincinnati, OH) and the TetO-CTGF mice were generated in the University of Miami Transgenic Facility. To generate double transgenic mice, the homozygous SP-C-rtTA mice were mated with the heterozygous TetO-CTGF mice. The newborn mice were genotyped by PCR of tail DNA with SP-C-rtTA and TetO-CTGF primers as previously described (33). The single transgenic SP-CrtTA mice were used as control mice, and the double transgenic SPC-rtTA/Teto-CTGF mice were used and referred to as CTGF+ mice. To induce CTGF expression in the lungs of newborn pups, the nursing dams were fed with Dox containing water $(1 \mathrm{mg} / \mathrm{ml})$ from postnatal day $(\mathrm{P}) 1$.

\section{Primary AT II Cell Culture}

AT II cells were isolated and cultured from 4 wk old control and CTGF+ mice as previously described with some modification $(33,40)$. Mice were sacrificed by intraperitoneal injection of pentobarbital and tracheotomized. The lungs were infused with dispase solution followed by low melting agarose via a tracheal catheter and were covered by ice to allow the agarose to polymerize. The lungs were then dissected and digested in dispase and teased from the bronchi in DNase solution. The cell suspension was filtered through gradient cell strainers $(100 \mu \mathrm{m}$ to $20 \mu \mathrm{m})$, and the cells were pelleted by centrifugation and then placed on tissue culture plates precoated with antiCD 45 and anti-CD 32 antibodies (BD Biosciences, San Jose, CA). After incubation for $2 \mathrm{~h}$ in a humidified $37^{\circ} \mathrm{C}$ incubator with $21 \% \mathrm{O}$ and $5 \% \mathrm{CO}_{2}$, the AT II cells (nonadhered) were collected, centrifuged and placed on Matrigel (70\%)-coated eight-well chamber slides or six-well plates (BD Biosciences). The AT II cells were cultured overnight in bronchial epithelial cell growth medium minus hydrocortisone (Lonza, Portsmouth, NH) with $5 \%$ charcoal stripped fetal calf serum and keratinocyte growth factor. The media were changed to fresh media containing Dox $(1 \mu \mathrm{g} / \mathrm{ml})$ and the cells were cultured for up to $96 \mathrm{~h}$. The purity of AT II cells was typically $>90 \%$, as assessed by pro-SP-C immunofluorescence staining.

\section{Knockdown of ILK}

A mix of three siRNA targeting mouse ILK (Qiagen, Valencia, CA) were used to knockdown ILK in AT II cells. These siRNA preparations $(100 \mathrm{nmol} / \mathrm{l}$ of each) or all-star negative control siRNA were incubated with HiPerFect (Qiagen) and then added to the AT II cells that have been cultured overnight according to the manufacture protocol. After $24 \mathrm{~h}$ transfection, the cell culture media was changed to fresh bronchial epithelial cell growth medium containing $1 \mu \mathrm{g} / \mathrm{ml}$ of doxycycline. The transfection was repeated at $48 \mathrm{~h}$ to increase transfection efficiency. At $96 \mathrm{~h}$, live cell morphology was imaged under a light microscope.

\section{Analysis of Gene Expression by qRT-PCR}

Total RNA was isolated from frozen lung tissues and AT II cells and treated with DNase to remove possible DNA contamination as described (34). One $\mu \mathrm{g}$ of total RNA was reverse-transcribed in a 20 $\mu \mathrm{l}$ reaction by using a first-strand cDNA synthesis kit according to manufacturer's protocol (Invitrogen, Grand Island, NY). The Realtime RT-PCR was performed on an ABI Fast 7500 System (Applied Biosystems, Foster City, CA). Each reaction included diluted firststrand cDNA, specific primers and master mix containing enzymes and TaqMan probes according to the manufacture's instruction (Applied Biosystems). Real-time RT-PCR conditions were $95{ }^{\circ} \mathrm{C}$ for $10 \mathrm{~min}$, followed by 40 cycles of $95^{\circ} \mathrm{C}$ for $15 \mathrm{~s}$ and $60{ }^{\circ} \mathrm{C}$ for $30 \mathrm{~s}$. RNase-free water was used as a negative control. For each target gene, a standard curve was established by performing a series of dilutions of the first-strand cDNA. The mRNA expression levels of target genes were determined from the standard curve and normalized to GAPDH.

\section{Western Blot Analysis}

Total protein was extracted from cells with a RIPA buffer according to manufacturer's protocol (Santa Cruz Biotechnology, Santa Cruz, CA). The protein concentrations were measured by bicinchoninic acid protein assay using a commercial kit from Pierce Biotechnology (Rockford, IL). Total proteins ( $50 \mu \mathrm{g} / \mathrm{sample}$ ) were fractionated by SDS-PAGE on 4-12\% Tris-glycine precast gradient gels (Invitrogen) and then transferred to nitrocellulose membranes (Amersham, Piscataway, NJ). The membranes were incubated overnight at $4{ }^{\circ} \mathrm{C}$ with respective primary antibodies and then incubated for $1 \mathrm{~h}$ at room temperature with horseradish peroxidase (HRP)-conjugated secondary antibodies. Antibody bound proteins were detected using enhanced chemiluminescent (ECL) chemiluminescence methodology (Amersham). Membranes were then stripped with $0.2 \mathrm{~N} \mathrm{NaOH}$ and re-incubated with primary antibodies reactive with a normalization protein, $\beta$-actin. The intensities of protein bands were quantified by Quantity One Imaging Analysis Program (Bio-Rad, Hercules, CA).

\section{Double Immunofluorescence Staining}

The following primary antibodies were used in immunostaining and immunofluorescence staining: a goat anti-CTGF antibody from Santa 
Cruz Biotechnology; a mouse anti- $\alpha$-SMA antibody from Sigma (St Louis, MI); a rabbit anti-pro-SP-C antibody from Chemicon (Temecula, CA); a rabbit anti-ILK antibody from Cell Signaling Technology (Danvers, MA). The AT II live cells were fixed with a mix of acetone and methanol (1:1), incubated with blocking buffer containing $2 \%$ bovine serum albumin in phosphate-buffered saline, and then incubated with primary antibodies in blocking buffer for $1 \mathrm{~h}$ at room temperature. The cells were incubated with AlexaFluor 488 and/or AlexaFluor 594 labeled secondary antibodies (Invitrogen, Carlsbad, CA) for $1 \mathrm{~h}$ at room temperature. After being washed with phosphate-buffered saline, the cells were counter stained with DAPI (VectorLab, Burlingame, CA) and mounted with glycerol.

\section{Data Presentation and Statistical Analysis}

Results are expressed as means \pm SD. Statistical analysis was performed by one-way ANOVA. A $P<0.05$ was considered significant.

\section{STATEMENT OF FINANCIAL SUPPORT}

This study was supported by funds from Project New Born and the Batchelor Award of University of Miami, Miami, FL.

Disclosure: There is no conflict of interest to disclose.

\section{REFERENCES}

1. Coalson JJ. Pathology of new bronchopulmonary dysplasia. Semin Neonatol 2003;8:73-81.

2. Noble PW, Barkauskas CE, Jiang D. Pulmonary fibrosis: patterns and perpetrators. J Clin Invest 2012;122:2756-62.

3. Scotton CJ, Chambers RC. Molecular targets in pulmonary fibrosis: the myofibroblast in focus. Chest 2007;132:1311-21.

4. Champam HA. Epithelial-mesenchymal interactions in pulmonary fibrosis. Annu Rev Physiol 2011;73:413-35.

5. Kage $\mathrm{H}$, Borok Z. EMT and interstitial lung disease: a mysterious relationship. Curr Opin Pulm Med 2012;18:517-23.

6. Ihn H. Autocrine TGF-beta signaling in the pathogenesis of systemic sclerosis. J Dermatol Sci 2008;49:103-13.

7. Kulozik M, Hogg A, Lankat-Buttgereit B, Krieg T. Co-localization of transforming growth factor beta 2 with alpha 1 (I) procollagen mRNA in tissue sections of patients with systemic sclerosis. J Clin Invest 1990;86:917-22.

8. Oi M, Yamamoto T, Nishioka K. Increased expression of TGF-betal in the sclerotic skin in bleomycin-'susceptible' mouse strains. J Med Dent Sci 2004;51:7-17.

9. Blobe GC, Schiemann WP, Lodish HF. Role of transforming growth factor beta in human disease. N Engl J Med 2000;342:1350-8.

10. Bonniaud P, Margetts PJ, Kolb M, et al. Progressive transforming growth factor beta1-induced lung fibrosis is blocked by an orally active ALK5 kinase inhibitor. Am J Respir Crit Care Med 2005;171:889-98.

11. Kolb M, Margetts PJ, Sime PJ, Gauldie J. Proteoglycans decorin and biglycan differentially modulate TGF-beta-mediated fibrotic responses in the lung. Am J Physiol Lung Cell Mol Physiol 2001;280:L1327-34.

12. Varga J, Pasche B. Transforming growth factor beta as a therapeutic target in systemic sclerosis. Nat Rev Rheumatol 2009;5:200-6.

13. Perbal B. CCN proteins: multifunctional signalling regulators. Lancet 2004;363:62-4.

14. de Winter P, Leoni P, Abraham D. Connective tissue growth factor: structure-function relationships of a mosaic, multifunctional protein. Growth Factors 2008;26:80-91.

15. Grotendorst GR. Connective tissue growth factor: a mediator of TGF-beta action on fibroblasts. Cytokine Growth Factor Rev 1997;8:171-9.

16. Kothapalli D, Frazier KS, Welply A, Segarini PR, Grotendorst GR. Transforming growth factor beta induces anchorage-independent growth of NRK fibroblasts via a connective tissue growth factor-dependent signaling pathway. Cell Growth Differ 1997;8:61-8.

17. Garrett Q, Khaw PT, Blalock TD, Schultz GS, Grotendorst GR, Daniels JT. Involvement of CTGF in TGF-beta1-stimulation of myofibroblast differentiation and collagen matrix contraction in the presence of mechanical stress. Invest Ophthalmol Vis Sci 2004;45:1109-16.

18. Duncan MR, Frazier KS, Abramson S, et al. Connective tissue growth factor mediates transforming growth factor beta-induced collagen synthesis: down-regulation by cAMP. FASEB J 1999;13:1774-86.
19. Gao R, Brigstock DR. Connective tissue growth factor (CCN2) induces adhesion of rat activated hepatic stellate cells by binding of its C-terminal domain to integrin alpha(v)beta(3) and heparan sulfate proteoglycan. J Biol Chem 2004;279:8848-55.

20. Heng EC, Huang Y, Black SA Jr, Trackman PC. CCN2, connective tissue growth factor, stimulates collagen deposition by gingival fibroblasts via module 3 and alpha6- and betal integrins. J Cell Biochem 2006;98:409-20.

21. Jedsadayanmata A, Chen CC, Kireeva ML, Lau LF, Lam SC. Activationdependent adhesion of human platelets to Cyr61 and Fisp12/mouse connective tissue growth factor is mediated through integrin alpha(IIb) beta(3). J Biol Chem 1999;274:24321-7.

22. Schwartz MA, Ginsberg MH. Networks and crosstalk: integrin signalling spreads. Nat Cell Biol 2002;4:E65-8.

23. Joshi MB, Ivanov D, Philippova M, Erne P, Resink TJ. Integrin-linked kinase is an essential mediator for T-cadherin-dependent signaling via Akt and GSK3beta in endothelial cells. FASEB J 2007;21:3083-95.

24. Naska S, Park KJ, Hannigan GE, Dedhar S, Miller FD, Kaplan DR. An essential role for the integrin-linked kinase-glycogen synthase kinase-3 beta pathway during dendrite initiation and growth. J Neurosci 2006;26: 13344-56.

25. Medici D, Nawshad A. Type I collagen promotes epithelial-mesenchymal transition through ILK-dependent activation of NF-kappaB and LEF-1. Matrix Biol 2010;29:161-5.

26. Fuchs BC, Fujii T, Dorfman JD, et al. Epithelial-to-mesenchymal transition and integrin-linked kinase mediate sensitivity to epidermal growth factor receptor inhibition in human hepatoma cells. Cancer Res 2008;68:2391-9.

27. Weaver MS, Toida N, Sage EH. Expression of integrin-linked kinase in the murine lens is consistent with its role in epithelial-mesenchymal transition of lens epithelial cells in vitro. Mol Vis 2007;13:707-18.

28. Sato S, Nagaoka T, Hasegawa M, et al. Serum levels of connective tissue growth factor are elevated in patients with systemic sclerosis: association with extent of skin sclerosis and severity of pulmonary fibrosis. J Rheumatol 2000;27:149-54.

29. Abraham D. Connective tissue growth factor: growth factor, matricellular organizer, fibrotic biomarker or molecular target for anti-fibrotic therapy in SSc? Rheumatology (Oxford) 2008;47:Suppl 5:v8-9.

30. Alapati D, Rong M, Chen S, et al. Connective tissue growth factor antibody therapy attenuates hyperoxia-induced lung injury in neonatal rats. Am J Respir Cell Mol Biol 2011;45:1169-77.

31. Sonnylal S, Shi-Wen X, Leoni P, et al. Selective expression of connective tissue growth factor in fibroblasts in vivo promotes systemic tissue fibrosis. Arthritis Rheum 2010;62:1523-32.

32. Sonnylal $\mathrm{S}, \mathrm{Xu} \mathrm{S}$, Jones $\mathrm{H}$, et al. Connective tissue growth factor causes EMT-like cell fate changes in vivo and in vitro. J Cell Sci 2013;126(Pt 10):2164-75.

33. Chen S, Rong M, Platteau A, et al. CTGF disrupts alveolarization and induces pulmonary hypertension in neonatal mice: implication in the pathogenesis of severe bronchopulmonary dysplasia. Am J Physiol Lung Cell Mol Physiol 2011;300:L330-40.

34. Liu BC, Li MX, Zhang JD, Liu XC, Zhang XL, Phillips AO. Inhibition of integrin-linked kinase via a siRNA expression plasmid attenuates connective tissue growth factor-induced human proximal tubular epithelial cells to mesenchymal transition. Am J Nephrol 2008;28:143-51.

35. Ivkovic S, Yoon BS, Popoff SN, et al. Connective tissue growth factor coordinates chondrogenesis and angiogenesis during skeletal development. Development 2003;130:2779-91.

36. Crosby LM, Waters CM. Epithelial repair mechanisms in the lung. Am J Physiol Lung Cell Mol Physiol 2010;298:L715-31.

37. Wynn TA. Common and unique mechanisms regulate fibrosis in various fibroproliferative diseases. J Clin Invest 2007;117:524-9.

38. Yao HW, Xie QM, Chen JQ, Deng YM, Tang HF. TGF-betal induces alveolar epithelial to mesenchymal transition in vitro. Life Sci 2004;76:29-37.

39. Willis BC, Liebler JM, Luby-Phelps K, et al. Induction of epithelial-mesenchymal transition in alveolar epithelial cells by transforming growth factor-beta1: potential role in idiopathic pulmonary fibrosis. Am J Pathol 2005;166:1321-32.

40. Yu W, Fang X, Ewald A, et al. Formation of cysts by alveolar type II cells in three-dimensional culture reveals a novel mechanism for epithelial morphogenesis. Mol Biol Cell 2007;18:1693-700. 